\title{
Changes of some anti-oxidative physiological indices under soil water deficits among 10 wheat (Triticum aestivum L.) genotypes at tillering stage
}

\author{
Hong-Bo Shao ${ }^{\text {a,b,c,*}}$, Li-Ye Chu ${ }^{\mathrm{c}}$, Gang Wu ${ }^{\mathrm{d}, \mathrm{b}, * *}$, Jin-Heng Zhang ${ }^{\mathrm{f}}$, \\ Zhao-Hua $\mathrm{Lu}^{\mathrm{a}, \mathrm{e}}$, Ya-Chen $\mathrm{Hu}^{\mathrm{g}}$ \\ ${ }^{a}$ Binzhou University ,Binzhou 256603, China \\ ${ }^{\mathrm{b}}$ Qingdao Institute of Biomass Energy and Bioprocess Technology, Chinese Academy of Sciences, Qingdao 266701, China \\ ${ }^{c}$ College of Molecular and Chemical Engineering, Qingdao University of Science and Technology, Qingdao 266042, PR China \\ ${ }^{\mathrm{d}}$ State Key Laboratory of Urban and Regional Ecology, Research Center for Eco-environmental Sciences, Chinese Academy of Sciences, Bejing100085, China \\ ${ }^{\mathrm{e}}$ Institute of Restoration Ecology, China University of Mining and Technology, Beijing 100083, PR China \\ ${ }^{\mathrm{f}}$ College of Environmental Sciences and Safety, Qingdao University of Science and Technology, Qingdao 266042, China \\ g Teaching Affairs Department, Jilin Normal University, Siping 136000, China
}

Received 15 July 2006; received in revised form 16 August 2006; accepted 7 September 2006

Available online 12 September 2006

\begin{abstract}
Drought is one of the major ecological factors limiting crop production and food quality globally, especially in the arid and semi-arid areas of the world. Wheat is the staple food for more than $35 \%$ of world population and wheat cultivation is mainly restricted to such zones with scarcity of water, so wheat anti-drought physiology study is of importance to wheat production, food safety and quality and biotechnological breeding for the sake of coping with abiotic and biotic conditions. The current study is to investigate changes of anti-oxidative physiological indices of 10 wheat genotypes at tillering stage. The main results and conclusion of tillering stage in terms of activities of POD, SOD, CAT and MDA content as followed: (1) 10 wheat genotypes can be generally grouped into three kinds (A-C, respectively) according to their changing trend of the measured indices; (2) A group performed better drought resistance under the condition of treatment level 1 (appropriate level), whose activities of anti-oxidative enzymes (POD, SOD, CAT) were higher and MDA lower; (3) B group exhibited stronger anti-drought under treatment level 2 (light-stress level), whose activities of anti-oxidative enzymes were higher and MDA lower; (4) C group expressed anti-drought to some extent under treatment level 3 (serious-stress), whose activities of anti-oxidative enzymes were stronger, MDA lower; (5) these results demonstrated that different wheat genotypes have different physiological mechanisms to adapt themselves to changing drought stress, whose molecular basis is discrete gene expression profiling (transcriptom). The study in this respect is the key to wheat anti-drought and biological-saving water in worldwide arid and semi-arid areas; (6) POD, SOD, and CAT activities and MDA content of different wheat genotypes had quite different changing trend at different stages and under different soil water stress conditions, which was linked with their origin of cultivation and individual soil water threshold, which will provide better reference to selecting proper plant species for eco-environmental construction and crops for sustainable agriculture in arid and semi-arid areas.
\end{abstract}

(C) 2006 Published by Elsevier B.V.

Keywords: Wheat; Drought resistance; Anti-oxidative stress; POD; SOD; CAT; MDA; Physiological mechanism; Soil water stress threshold; Water-saving biology; Arid and semi-arid areas

\footnotetext{
Abbreviations: FC, field capacity; POD, peroxidase; SOD, superoxide dismutase; CAT, catalase; MDA, malondialdehyde

* Corresponding author at: Biology Department, College of Molecular and Chemical Engineering, Qingdao University of Science and Technology, Qingdao 266042, PR China.

** Corresponding author.

E-mail addresses: shaohongbochu@126.com (H.-B. Shao), wugang@qibebt.ac.cn (G. Wu).
} 


\section{Introduction}

Drought is a worldwide problem, constraining global crop production seriously $[1,2,5,7,19-21,37,45,46,59]$ and recent global climate change has made this situation more serious [15,30,32,35,38,64]. Drought is a complex physical-chemical process, in which many biological macromolecules and small molecules are involved, such as nucleic acids (DNA, RNA, microRNA), proteins, carbohydrates, lipids, hormones, ions, free radicals, mineral elements $[3,8,12,13,18,20,22,26,31,43,49,50,55,65]$. In addition, drought is also related to salt stress, cold stress, high temperature stress, acid stress, alkaline stress, pathological reactions, senescence, growth, development, cell cycle, UV-B damage, wounding, embryogenesis, flowering, signal transduction and so on $[16,17,26-28,36,37,44-47,52-54,62]$. Therefore, drought is connected with almost all aspects of biology. Currently, drought study has been one of the main directions in global plant biology and biological breeding. NSF in USA established the program of Plant Genome Functions Under Stresses in 1998, and strengthened it in 2000, and drafted it towards 2010 [40,41]. Just 2 years ago, European Commission, who once kept conservative to biotechnological breeding, constructed a big project: Plants for the Future in which much is involved in resistance drought [5]. Many advances in relation to this hot topic, including molecular mechanism of antidrought and corresponding molecular breeding have taken place [4,10,16,17,21,23-25,30,31,35,37,39,42,48,55-59]. Although the obtained transgenic crops (mainly, wheat) by different types of gene technology all exhibit resistance drought to some extent, they have many shortfalls related to agronomical performance and/or development $[1,16,17,21,24,30,39,49,56]$. The partial reason is that wheat drought resistance character is a quantitative one controlled by multi-genes in a bigger genome $(16,000 \mathrm{Mb})$. These results also imply that systemical, deeper, and comprehensive understanding of physiological mechanism of crops under drought stresses is not enough to manipulate the physiological regulatory mechanism and take advantage of full this potential for productivity, Whose study is the bridge between molecular machinery of drought and anti-drought agriculture, because the performance of genetic potential of crops is expressed by physiological realization in fields $[36,53,56,62,65]$. Towards this aim, many promising methodologies emerge, but they should also be linked with field practice $[6,9,11,14,25,42,54,57]$.

Wheat (Triticum aestivum L.) is a staple food for more than $35 \%$ of the world population and wheat is also the second grain crop in China, whose production status is directly related to social stability, Chinese survival and sustainable development $[52,60,61]$. In China, the cultivation area for wheat has reached $3.12 \times 10^{5} \mathrm{hm}^{2}$ and the total wheat production has been more than $1.04 \times 10^{6} \mathrm{t}$, which is the biggest country for wheat production and supply on the globe [61]. With progressive global climate change and increasing shortage of water resources and worsening eco-environment, wheat production is influenced greatly $[7,15,29,32-34,38,41,51,58,64]$. Anti-oxidative performance in higher plants have been thought to be one of the main physiological mechanisms and be central in responses to soil water deficits $[12,27,28,47,52,53]$. POD (peroxidase), SOD (superoxide dismutase) and CAT (catalase) are main components of anti-oxidative machinery for drought resistance in higher plants. MDA (malondialdehyde) content is one main parameter for evaluating membrane oxidation extent [12,52]. To aim at making different wheat genotypes perform fully physiological potential under limited soil water conditions in fields, selecting practical materials for direct breeding and establishing an efficient platform for deciphering molecular mechanisms of wheat drought resistance, especially, the link of the redox state with anti-drought, and for agricultural sustainable development of arid and semiarid regions on the globe, this experiment was conducted.

\section{Materials and methods}

\subsection{Plant materials}

Ten wheat genotypes (Xinong9-1-1-13, Xinong-1, Xinong32, Xinong4-2, Xinong9337-1, YB0738, Xiaobingcao7, Jinmai47, Yumai49, Xiaoyan22, and labeled 1-10, respectively) were provided by Yangling Breeding Center of National Wheat Engineering Research Center of China. Jinmai47, yumai49 and Xiaoyan22 (8-10, respectively) have been identified as better cultivars for being popularized in different locations of China. The other seven wheat genotypes are promising ones in practice (field experiments have proved).

\subsection{Experimental fields}

The outward potting cultivation field is affiliated to experimental plots of National Key Laboratory of Soil Erosion and Dryland Farming, the Center of Soil and Water Conservation and Eco-environmental Research, Chinese Academy of Sciences. Pots are made of black plastics, whose empty weight is $2 \mathrm{~kg}$ and filled-soil weight $24 \mathrm{~kg}$ according to Gao [63]. The selected soil was from the farmland in the suburbs of Yangling Model District, Shanxi, China. The basic property of physics and chemistry from the selected soil as follows: organic matter $11.2 \mathrm{mg} / \mathrm{g}$, total $\mathrm{N} 0.93 \mathrm{mg} / \mathrm{g}$, available N $122.1 \mathrm{mg} / \mathrm{kg}$, effective P $52.2 \mathrm{mg} / \mathrm{kg}$, available $\mathrm{K} 233.2 \mathrm{mg} / \mathrm{kg}$.

\subsection{Experimental design}

Pot-cultivating experiments were carried out outdoors and stimulation natural drought method was selected. Each genotype was conducted in three level soil water treatments ( $75 \% \mathrm{FC}, 55 \%$ $\mathrm{FC}$, and $45 \% \mathrm{FC}$, respectively) controlled by weighting method, each of which is six times repeated and matched with one control, correspondingly. All the data from measurement were averaged from the data for six times.

\subsection{Collection and measurement of anti-oxidative enzymes}

SOD (EC 1.1.5.1.1), POD (EC 1.11.1.7), CAT (EC 1.11.1.6) measurement of wheat tillering stage was conducted according to Zhang and Kirham [62] and Shao et al. [53]. Fresh flag 
leaves $(0.5 \mathrm{~g})$ was well homogenized, respectively, with extraction buffers (phosphate acid buffer $\mathrm{pH}$ 7.8) and centrifuged for $15 \mathrm{~min}$ at $10,000 \times g 4^{\circ} \mathrm{C}$. The supernatant was collected, respectively, for measurement of corresponding absorption by UV-vis spectrophotometer (Tongfang Inc., Shanghai, China) as described above. MDA (malondialdehyde) detecting was done by referring to Gao [63].

\section{Results}

\subsection{POD activities of 10 wheat genotypes at tillering stage}

From Fig. 1, genotypes 2, 8-10 (A group) expressed higher POD activities under level 1; genotypes 1, 3, 4, 6, 7 (B group) had stronger POD activities at level 2; genotype 5 (C group) possessed relatively higher POD activities under the condition of level 3. Among A group, genotype 2 had the highest POD activities (about 800 units); among B group, genotype 6 expressed the strongest POD activities (about 1100 units); genotype 5 of C group had roughly 780 units of POD activities. Comparing A-C groups under different soil water levels, we found that genotype 6 had the highest POD activities, showing that this wheat genotype performed higher drought resistance under level 2. In addition, of A group, genotypes 8-10 expressed little difference under three treatment levels, demonstrating that genotype 2 performed a contrasting characteristic, i.e. under levels 1 and 2 , it had little difference, but at level 3 , it had a great reduction in POD activities. This implied that the former three genotypes were insensitive to soil water stress to some extent, had similar soil water stress threshold, and better drought resistance, which was liked with the location of cultivation and selection. Genotype 2 had more sensitive ability, in particular, at level 3, expressed the lowest POD activities of all 10 wheat genotypes (about 100 units) irrespective of treatment levels, reflecting lower drought resistance and higher anti-drought threshold in comparison with the other genotypes of A group. Among B group, genotypes 1, 3, 6,7 expressed similar changing trend, in which they had higher POD activities under levels 1 and 2 and lower POD activities under level 3 , showing that soil water of level 2 was probably appropriate for these wheat genotypes. These genotypes could not perform better drought resistance under level 1 (relative water-logging), especially for genotype 4 . The reason for higher expression of POD activities in genotypes 6 and 7 (B group) than those of genotypes 2, 8-10 (A group) might be due to their genetic background difference (discrete alleles) and dif-

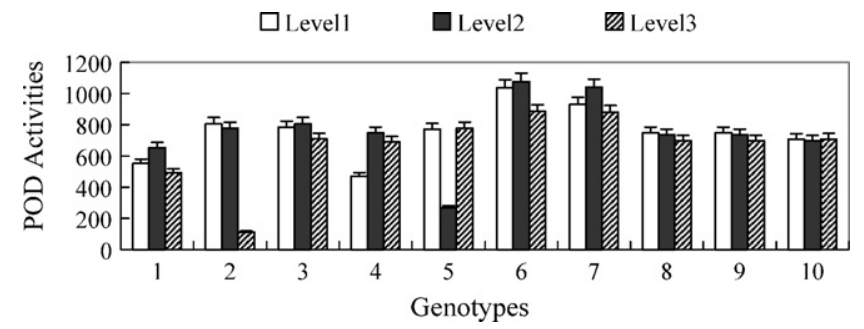

Fig. 1. POD activities of 10 wheat genotypes at tillering stage. Vertical bars represent standard errors $(n=6)$.

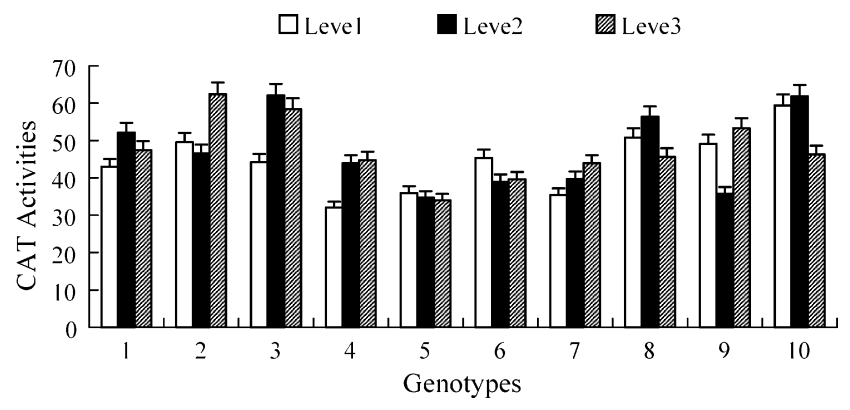

Fig. 2. CAT activities of 10 wheat genotypes at tillering stage. Vertical bars represent standard errors $(n=6)$.

ferent generations to be cultivated (see Section 4). Overall results showed that different wheat genotypes had different anti-drought threshold and the status of redox in wheat different genotypes were exist with their life cycle and changed with their settings.

\subsection{CAT activities of 10 wheat genotypes at tillering stage}

According to Fig. 2, we could classify genotypes 5, 6 into A group, which had stronger CAT activities at level 1, genotypes $1,3,8,10$ into B group, expressing higher CAT activities under level 2, genotypes 2, 4, 7, 9 into $C$ group, which performed higher CAT activities at level 3. Comparing A-C group, genotypes 2, 3, 10 had the highest CAT activities (about 63 units) of these three treatment levels. Between A group, genotypes 5 and 6 had no obvious difference and similar changing trend under levels 2 and 3, in particular, for genotype 5 almost with the same CAT activities at levels $1-3$, showing that both of them had lower ability to respond to reducing soil water content in terms of CAT activity change. Among B group, genotypes 1 and 3 had the same changing character, i.e. CAT activities at level 3 were bigger than those at level 1, showing that these two genotypes had active responses to a certain water stress; genotypes 8 and 10 had similar phenotype, i.e. the activities of CAT of these two genotypes at level 1 were stronger than those at level 3, demonstrating that genotypes 8 and 10 could not express better anti-drought under water-limited conditions. Among $\mathrm{C}$ group, we could find that genotypes 4 and 7 expressed increasing CAT activities with soil water stress strength, implying that these two genotypes had better drought resistance and lower anti-drought threshold, which also provided physiological machinery for both drought breeding and water-saving agriculture.

\subsection{SOD activities of 10 wheat genotypes at tillering stage}

From Fig. 3, it was observed that genotypes 1, 2, 4, 6, 8, 9 (A group) had higher SOD activities under level 1, genotypes 3, 5, 7 (B group) had stronger SOD activities at level 2, and genotype 10 (C group) had higher SOD activities under level 3 . By comparing A-C group, we found that genotype 2 had the strongest SOD activities (nearly 440 units) at level 1 in all treatment levels and genotypes. Among A group, genotypes 1, 2, 4 expressed the same changing trend, in which their SOD activities reduced with soil water stress strength, showing their lower ability to respond to water-deficiency conditions. Geno- 


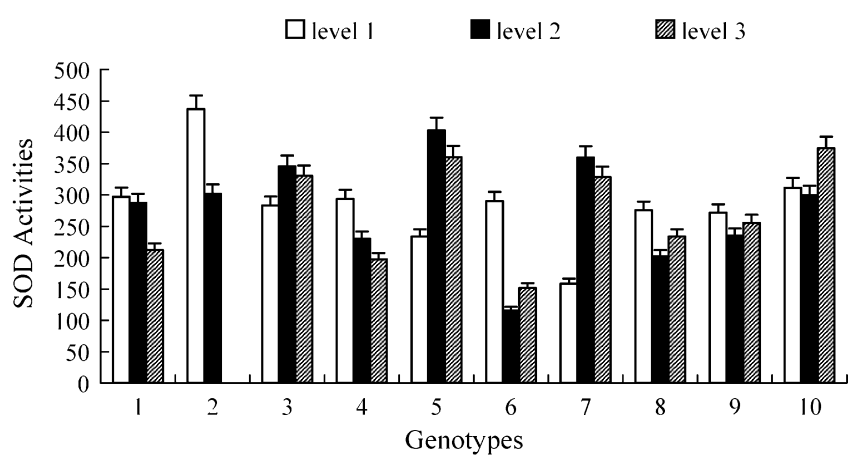

Fig. 3. SOD activities of 10 wheat genotypes at tillering stage. Vertical bars represent standard errors $(n=6)$.

types 6, 8, 9 expressed a different pattern, i.e. the SOD activities of these three genotypes at level 3 were bigger than those at level 2, implying that they had lower anti-drought threshold, better resistance drought, and their corresponding SOD droughtinducible gene expression was performed fully at severe water deficits (level 3). These results provided a new clue to selecting drought-inducible genes and further study of wheat anti-drought nature. Among B group, all members had the similar changing format that the SOD activities at level 3 were higher than those at level 1 and expressed similar fluctuations. This demonstrated that SOD gene expression of these genotypes could be realized fully under level 1 (relative water-logging) and performed better at level 3, implying that anti-drought threshold (water stress threshold) was possibly an important trigger to initiate SOD drought-inducible genes. The SOD activities of genotype 10 of $\mathrm{C}$ group also prove this presumption.

\subsection{MDA content of 10 wheat genotypes at tillering stage}

According to Fig. 4, genotypes 1, 4, 5, 9, 10 (A group) had lower MDA content under level 1, reflecting better membrane integrity, genotype 7 (B group) had lower MDA content under level 2, genotypes 2, 3, 6, 8 (C group) had lower MDA content at level 3, showing that these genotypes expressed better membrane completeness, further exhibiting stronger resistance

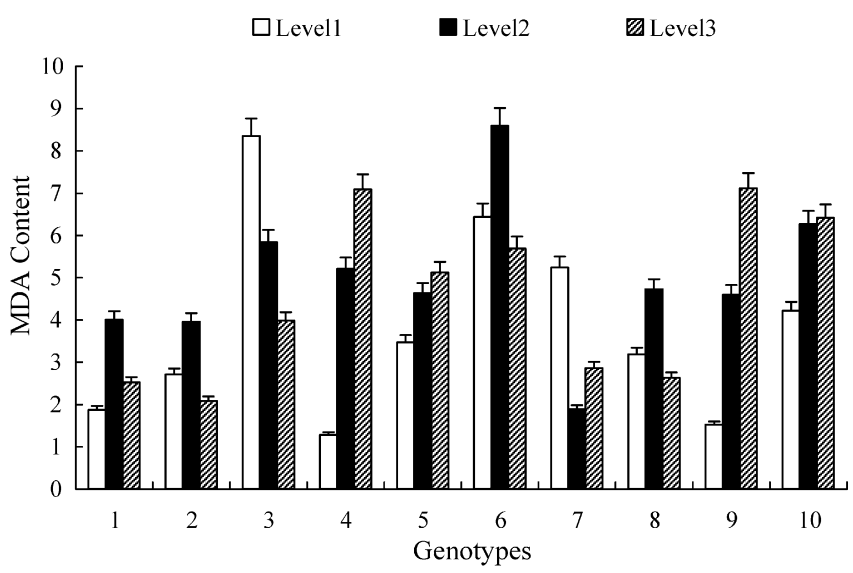

Fig. 4. MDA content of 10 wheat genotypes at tillering stage. Vertical bars represent standard errors $(n=6)$. drought under serious soil water stressed conditions. Comparing A-C group, genotype 4 had the lowest MDA content (about 1.2 units) among 10 genotypes, but it could not perform good anti-drought with increasing degree of soil water stress, implying that it had a higher soil water stress threshold. Among A group, genotypes 4, 5, 9, 10 had the same trend of MDA content reflected from their individual treatment levels (from levels 1 to 3), i.e. MDA content increased with progressive stressing course, implying that these genotypes could not maintain the status of membrane anti-oxidation when soil water content was not enough to reach "so-called appropriate level" and had lower anti-drought in the sense of MDA content. Genotype 7 of B group had the highest MDA content at level 1, higher at level 3 and the lowest at level 2, showing that the soil water of level 2 might be its appropriate level of this genotype, and predesigned level 1 was not. So, the fact and method accepted and adopted by most scholars that $75 \%$ FC, $55 \%$ FC, and $45 \%$ FC is appropriate, light-stressed, and serious-stressed, respectively, was doubted. Among C group, genotypes 2, 6, 8 had similar pattern of MDA content change, i.e. MDA content at level 1 was higher than that at level 3 and the MDA content at level 2 was the highest of these three levels, showing that the genes involved in MDA metabolism in these genotypes were started to stronger extent at level 1 and their expression reached a peak at level 2, in the process of which other protective mechanisms began with participation and made MDA content reduce to be the lowest at level 3 . These results implied that many events were involved in protecting membrane systems of wheat different genotypes from oxidation when they encounter with soil water stress.

\section{Discussion}

Plants have evolved a wide range of defense systems to survive continuous assault by an arsenal of biotic attacks, constantly changing weather and other environmental conditions [42,55,44-46,16,21,48,36] (Cushman and Bohnert, 2000). Unraveling the physiological and molecular basis for the plasticity of plant defending metabolism not only provides access to a largely untapped resource of genes and selection markers for breeding enhanced stress tolerance in crops but also ensures improved food security world-wide [64,23,16,12,2,5,20,24,32,34,62,50,52,54] (Fricke et al., 2004; Trono et al., 2002).

Living organisms can be viewed as reducing-oxidising (redox) systems in which catabolic, largely oxidative processes produce energy and anabolic, principally reductive processes assimilate it $[13,27,28,47]$. Aerobic organisms exploit the redox potential of oxygen while controlling oxidation. A key feature determining the size of the plant "physiological window" where metabolic functions can be maintained and regulated, is the extent to which oxidative reactions can be tightly controlled [12]. If environmental changes are too extreme to allow short-term metabolic controls to maintain fluxes through primary metabolism while preventing uncontrolled oxidation, then stress induced damage ensues. Here, there is an obvious stress threshold [12,51-53]. In this situation acclamatory changes in 
gene expression are induced in attempt to restore redox homeostasis $[46,42,55]$. If the repertoire of genomic responses is not insufficient or not appropriate then primary metabolism is impaired, oxidative stress becomes increasingly important and cell death and senescence responses are triggered [13,27,28] (Harding et al., 2003). One of the earliest responses of plants to pathogens, wounding, drought, extremes of temperature or physical and chemical shocks is the accumulation of active oxygen species (AOS) such as superoxide, hydroxyl radicals, hydrogen peroxide and singlet oxygen [47]. The oxidative stress that ensues is a widespread phenomenon [46-48]. It is observed in wheat exposed to most, if not all, biotic and environmental stresses. AOS are key components contributing to cellular redox poise. They participate in all processes controlled by redox reactions. These include signal transduction, gene expression, protein synthesis and turnover, thiol-disulphide exchange reactions and regulation of metabolism. AOS accumulation is sensed as an 'alarm' signal that initiates pre-emptive defense responses [15,20,38,2,54,42,55,51,3] (Shao et al., 2004a). Common and linked signal transduction pathways are activated that can lead either to stress acclimation or to cell death depending on the degree of oxidative stress experienced. Wheat responses to stresses are therefore directed to acclimate and repair damage, which is the basic common feature of organisms [18,34,32,36] (Harding et al., 2003).

POD, SOD, CAT and MDA are common and important indices for evaluating the status of wheat redox, whose activities of the former 3 and whose lower of MDA display higher anti-oxidative ability, reflecting higher resistance drought $[7,13,15,24,34,62]$. SOD (superoxide dismutase) is a type of enzymes with metals, whose function is to get rid of oxygen free radicals or others and protect membrane systems. POD (perioxidase) are enzymes that catalyze polyphenolic compounds into other products. CAT (catalase) is the enzyme that scanavenges $\mathrm{H}_{2} \mathrm{O}_{2}$ in cells [12,27,28]. In our experiment, our results showed that different wheat genotypes clearly responded to soil water deficiency differently in terms of activities of POD, SOD, CAT and MDA content (Figs. 1-4). Erdei et al. [59] selected Hungarian wheat cultivars as materials and analyzed their physiological responses to soil water deficits. They found that drought resistance in different cultivars was significantly different $(p<0.05)$. Our results were in good agreement with this. Dhanda et al. [7] measured drought resistance parameters from 19 wheat genotypes at the early stage of growth and they concluded that anti-oxidative indices (SOD, POD, CAT, MDA and so on) were important to evaluate drought resistance among different wheat genotypes. This further implied that different wheat genotypes had discrete water stress threshold and therefore they had different physiological adaptive mechanisms to regulate their redox status [47,51-53,67]. Obviously, the difference in this aspect exhibited the difference in their alleles of different wheat genotypes [2,15,59] (Saranga et al., 2002). The emerging methodologies including, genomic approaches, system-oriented ways, large-scale microarray technology, genome-metabolite profiling analysis and transcriptom profiling precise annotation, in combination with genetic mutant selection, transgenic strategy and plant physiology, will uncover this network regulatory system of wheat genotypes being adaptive to different soil water stresses [26,36,38,44-46,66,67].

Generally, our results firstly clearly showed that different wheat genotypes differently responded to soil water stress at different stages in terms of physiological mechanisms, implying that they had different soil water stress thresholds [52]. Exploring its range and accurate amount of different genotypes is of importance to understanding physiological mechanisms of wheat resistance and tolerance drought and saving-water agriculture by the way of physiological regulation. Secondly, our experimental results further demonstrated the fact accepted and adopted by most scholars, that $75 \% \mathrm{FC}, 55 \% \mathrm{FC}$, and $45 \% \mathrm{FC}$ is normal, light-stressed, and severe-stressed water level, respectively, is needed to be modified in order to represent the practical level of more plants [65]. Thirdly, the state of redox is exist with plant circle, but quite different at different stages and under the conditions of different soil water stresses [33,36,52]. In addition, POD, SOD, CAT and MDA have different changing time course, whose molecular basis is different expression-regulation machinery of corresponding genes, and cannot be regarded as only one criteria in evaluating wheat anti-drought. Fourthly, we also found that the changing trend of POD, SOD, and CAT activities and MDA content under our condition of three stress levels is tightly linked with the location of their cultivation, which reflects the change of corresponding alleles of different genotypes under the pressure of natural and artificial selection [61,62]. Knowing the refine physiological nature, in conjunction with natural rainfall status of different locations, is very important to popularize new wheat species and conduct wheat breeding.

In a word, the study of physiological mechanisms of wheat anti-drought has much work to do. Molecular biology aspects of wheat cannot substitute for this important part, but strengthen the research and provide a broad future. It is easy to see that one cell or molecule cannot be alive in natural fields and not provide any economic effect for human being. The combination of molecular biology and plant physiology is the key. Many achievements in biotechnological and traditional breeding of wheat are good examples [1,4,21,38,56,66,67]. Although some progresses in terms of the exploration of molecular nature of wheat anti-drought also have taken place, many problems exist. What is the relationship of mineral elements (in particular, $\mathrm{K}^{+}$ $\mathrm{Na}^{+}$) with root signal transduction (pathways)? Much of former study showed that $\mathrm{K}^{+}$was little connected with anti-drought, but recent research and our results displayed that it was linked with wheat resistance drought $[8,22,49,46,42,55]$. What is the exact soil water stress threshold of individual wheat genotype? This is of much importance to resistance drought breeding and savingagriculture and precise agriculture under global climate change. What are the details that constitute the network regulatory system of drought, cold, UV-B, freezing, acidity, salty, wounding, pathogen, senescence, cell death? How is each linked with other parts? What is the (transient) connection among different physiological adaptive regulatory pathways at different levels? What role do endogenous hormones play in this course? What is the crosstalk among them when abiotic or/and biotic stress happens? The redox state in plants is important, and how is it regulated? What is the relationship with microRNAs? A widespread use 
of data resources for fine gene functions and structure of different plants (species) is from model plants, Arabidopsis thaliana and rice, and how large is the reliability? No doubt, expanded detecting of plant range is more urgent. So, we think that physiological studies at different scales have much work to do with the increasing atmospheric change.

\section{Acknowledgements}

This work is jointly supported by Initiation Foundation of Qingdao University of Science and Technology (0022221) (H.B. Shao), Natural Science Foundation of China (No. 40473054) (G. Wu), (No. 70273050; 20176019) (Z.H. Lu) and (No. 40601062) (J.H. Zhang) Province, and Initiation Specialized Foundation of Excellent Doctoral Dissertation of Chinese Academy of Sciences. National Science and Technology Supporting Program (2006BAC15B03) (H.B. Shao). Many thanks are given to our post-graduate students, Mr. H.L. Zhuang, F.L. Chen, M. Zhou, X.R. Kong, Ms. J. Zhang, J.F. Yu, and Y.J. Shong for their help in field experiments and partial detesting. Special thanks were given to Prof. Peter J. Lillford in University of York, UK for his critically reading the manuscript and pointing out better suggestions and making instructive comments.

\section{References}

[1] D. Patnaitk, P. Khurana, Wheat biotechnology: a minireview, Electron. J. Biotechnol. 4 (2) (2001) 74-102.

[2] C. Glombitza, P.H. Dubuis, O. Thulke, Crosstalk and differential response to abiotic and biotic stressors reflected at the transcriptional level of effector genes from secondary metabolism, Plant Mol. Biol. 51 (2004) 1-19 (uncorrected proof).

[3] P. Casati, V. Walbot, Rapid transcriptome responses of maize (Zea mays) to UV-B irradiated and shielded tissues, Genome Biol. 5 (2004) R16.

[4] E. Stokstad, Monsanto pulls the plug on genetically modified wheat, Science 304 (5674) (2004) 1088-1089.

[5] European Commission, Plants for the future: a European Vision for Plant Genomics and Biotechnology towards 2025, 2004 (see the web site: http://www.europabio.org/).

[6] J. Kopka, A. Fernie, W. Weckwerth, Metabolite profiling in plant biology: platforms and destinations, Genome Biol. 5 (2004) 109-125.

[7] S.S. Dhanda, G.S. Sethi, R.K. Behl, Indices of drought tolerance in wheat genotypes at early stages of plant growth, J. Agron. Crop Sci. 190 (1) (2004) 6-12.

[8] F.J.M. Maathuis, V. Filatov, P. Herzyk, Transcriptom analysis of root transporters reveals participation of multiple gene families in the response to cation stress, Plant J. 35 (2003) 675-692.

[9] A. Rellegrineschi, J.M. Ribaut, R. Trethowan, Looking beyond the details: a rise in system-oriented approaches in genetics and molecular biology, Curr. Genet. 41 (1) (2002) 1-10.

[10] M.A. Villalobos, D. Bartels, G. Iturringa, Stress tolerance and glucose insensitive phenotypes in Arabidopsis overexpressing the CpMYB10 transcription factor gene, Plant Physiol. 135 (2004) 309-324.

[11] P. Wenzl, J. Carling, D. Kudrma, Diversity arrays technology (DArT) for whole-genome profiling of barley, PNAS 101 (26) (2004) 9915-9920.

[12] K. Apel, H. Hirt, Reactive oxygen species: metabolism, oxidative stress, and signal transduction, Annu. Rev. Plant Biol. 55 (2004) 373-399.

[13] Z. Chen, D.R. Gallie, The ascorbic acid redox state controls guard cell signaling and stomatal movement, Plant Cell 16 (2004) 1143-1162.

[14] S. Chris, S. Shauna, Plant functional genomics, Science 285 (1999) 380-383.

[15] J. Saba, M. Moghaddam, K. Ghassemi, Genetic properties of drought resistance indices, J. Agric. Sci. Technol. 3 (2001) 43-49.
[16] H. Wang, Z. Huang, Q. Chen, Ectopic overexpression of tomato JERF3 in tobacco activates downstream gene expression and enhances salt tolerance, Plant Mol. Biol. 51 (2004) 1-10 (uncorrected proof).

[17] T. Puhakainen, M.W. Hess, P. Makela, Overexpression of multiple dehydrin genes enhances tolerance to freezing stress in Arabidopsis, Plant Mol. Biol. 51 (2004) 1-11 (uncorrected proof).

[18] C. Delessert, I.W. Wilson, D. Van Der Straiten, Spatial and temporal analysis of the local response to wounding in Arabidopsis leaves, Plant Mol. Biol. 51 (2004) 1-18 (uncorrected proof).

[19] M. Kern, Food, feed, fibre, fuel and industrial products of the future: challenges and opportunities. Understanding the strategic potential of plant genetic engineering, J. Agric. Crop. Sci. 188 (5) (2002) 291-302.

[20] R. Munns, Comparative physiology of salt and water stress, Plant Cell Environ. 25 (2) (2002) 239-252.

[21] W.X. Wang, B. Vinocur, A. Altman, Plant responses to drought, salinity and extreme temperatures: towards genetic engineering for stress tolerance, Planta 218 (1) (2003) 1-14.

[22] A. Costa, A. Carpaneto, S. Varotto, Potassium and carrot embryogenesis: are $\mathrm{K}^{+}$channels necessary for development? Plant Mol. Biol. 51 (2004) 1-16 (uncorrected proof).

[23] Y.S. Liu, S. Roof, Z.B. Ye, Manipulation of light signal transduction as a means of modifying fruit nutritional quality in tomato, PNAS 101 (26) (2004) 9897-9902.

[24] T. Capell, L. Bassie, P. Christou, Modulation of the polyamine biosynthetic pathway in transgenic rice confers tolerance to drought stress, PNAS 101 (26) (2004) 9909-9914.

[25] M.Y. Hiral, M. Yano, D.B. Goodenowe, Integration of transcripomics and metabolomics for understanding of global responses to nutritional stresses in Arabidopsis, PNAS 101 (26) (2004) 1-6.

[26] X.A. Liu, W.M. Vance Baird, Identification of a novel gene, HAABRC5, from Helianthus annuus (Asteraceae) that is upregulated in response to drought, salinity, and abscisic acid, Am. J. Bot. 91 (2004) 184 191.

[27] S. Llesuy, P. Evelson, A.M. Campos, Methodologies for evaluation of total antioxidant activities in complex mixtures. A critical review, Biol. Res. 34 (2) (2001) 47-65.

[28] I. Urquiaga, F. Leighton, Plant polyphenol antioxidants and oxidative stress, Biol. Res. 33 (2) (2000) 59-75.

[29] Z. Plaut, Crop plants: critical development stages of water, Encyclopedia Water Sci. (2003) 95-100.

[30] A. Anand, H.N. Trick, B.S. Gill, Stable transgene expression and random gene silencing in wheat, Plant Biotechnol. J. 1 (4) (2003) 241-251.

[31] M.W. Jones-Rhoades, D.P. Bartel, Computational identification of plant MicroRNAs and targets including a stress-induced miRNA, Mol. Cell 14 (2004) 787-799.

[32] D.A. White, Drought, Encyclopedia Water Sci. (2003) 160-162.

[33] J.M. Morgan, Plants: osmotic adjustment, Encyclopedia Water Sci. (2003) 692-695.

[34] J.W. Chandler, D. Bartels, Drought avoidance and drought adaptation, Encyclopedia Water Sci. (2003) 163-165.

[35] H.X. Shou, P. Bordallo, K. Wang, Expression of the Nicotiana protein kinase (NPK1) enhanced drought tolerance in transgenic maize, J. Exp. Bot. 55 (399) (2004) 1013-1019.

[36] M.M. Chaves, J. Maroco, J. Pereira, Understanding plant responses to drought-from genes to the whole plant, Funct. Plant Biol. 30 (2003) 239-264.

[37] E. Mantyla, V. Lang, T. Palva, Role of abscisic acid in drought-induced freezing tolerance, cold acclimation, and accumulation of LT178 and RAB18 proteins in Arabidopsis, Plant Physiol. 107 (1995) 141-148.

[38] I. Yordanov, V. Velikova, T. Tsonev, Plant responses to drought and stress tolerance, Bulg. J. Plant Physiol. (Special Issue) (2003) 187-206.

[39] M. Kasuga, Q. Liu, S. Miura, Improving plant drought, salt, and freezing tolerance by gene transfer of a single-inducible transcription factor, Nat. Biotechnol. 17 (3) (1999) 287-291.

[40] C. Somerville, J. Dangl, Plant biology in 2010, Science 290 (4599) (2000) 2077-2078.

[41] Editor's Choice, The national plant genomics initiative: objectives for 2003-2008, Plant Physiol. 130 (2002) 1741-17449. 
[42] T. Zhu, Global analysis of gene expression using GeneChip microarrays, Curr. Opin. Plant Biol. 6 (5) (2003) 418-425.

[43] S.A. Casson, K. Lindsey, Genes and signaling in root development, N. Phytol. 158 (1) (2003) 11-34.

[44] K. Shinozaki, E.S. Dennis, Cell signaling and gene regulation global analyses of signal transduction and gene expression profiles, Curr. Opin. Plant Biol. 6 (5) (2003) 405-409.

[45] K. Shinozaki, K. Yamaguchi-Shinozaki, M. Seki, Regulatory network of gene expression in the drought and cold stress responses, Curr. Opin. Plant Biol. 6 (5) (2003) 410-417.

[46] J.K. Zhu, Salt and drought stress signal transduction in plants, Annu. Rev. Plant Biol. 53 (2002) 247-273.

[47] M.Y. Jiang, J.H. Zhang, Abscisic acid and antioxidant defense in plant cells, Acta Bot. Sin. 46 (1) (2004) 1-9.

[48] S.W. Yu, K.X. Tang, MAP kinase cascades responding to environmental stress in plants, Acta Bot. Sin. 46 (2) (2004) 127-136.

[49] F.Y. Zhao, S.L. Guo, Z.L. Wang, H. Zhang, Recent advances in study on transgenic plants for salt tolerance, J. Plant Physiol. Plant Mol. Biol. 29 (3) (2003) 171-178.

[50] H.B. Shao, Z.S. Liang, M.A. Shao, B.C. Wang, Impacts of PEG-6000 pretreatment for barley (Hordeum vulgare L.) seeds on the effect of their marure embryo in vitro culture and primary investigation on its physiological mechanism, Colloids Surf. B: Biointerfaces 41 (2) (2005) 73-77.

[51] H.B. Shao, Z.S. Liang, M.A. Shao, Roles of ABA signal transduction during higher plant seed maturation and germination, Forest. Stud. Chin. 5 (4) (2003) 42-51.

[52] H.B. Shao, Z.S. Liang, M.A. Shao, B.C. Wang, Changes of some physiological and biochemical indices for soil water deficits among 10 wheat genotypes at seedling stage, Colloids Surf. B: Biointerfaces 42 (1) (2005) 107-113.

[53] H.B. Shao, Z.S. Liang, M.A. Shao, Changes of some anti-oxidative enzymes under soil water deficits among 10 wheat genotypes at maturation stage, Colloids Surf. B: Biointerfaces 45 (4) (2005) 7-13.
[54] J.A. Kreps, Y.J. Wu, H.S. Chang, Transcriptome changes for Arabidopsis in response to salt, osmotic, and cold stress, Plant Physiol. 130 (2002) 2129-2141.

[55] J.K. Zhu, Regulation of ion homeostasis under salt stress, Curr. Opin Plant. Biol. 6 (5) (2003) 441-445.

[56] A. Pellegrineschi, J.M. Ribaut, R. Trethowan, K. Shinozaki, Progress in the genetic engineering of wheat for water-limited conditions, JIRCAS Working Report 55-60, 2002.

[57] J.C. Cushman, J. Bohnert, Genomic approaches to plant stress tolerance, Curr. Opin. Plant Biol. 3 (2) (2003) 117-124.

[58] M. Zhiponova, L. Szilaz, L. Erdei, Comparative approach for the isolation of genes involved in the osmotolerance of wheat, in: Proceedings of the Seventh Hungarian Congress on Plant Physiology, 2002, pp. S1-P11.

[59] L. Erdei, I. Tari, J. Csiszar, Osmotic stress responses of wheat species and cultivars differing in drought tolerance: some interesting genes (advices for gene hunting), Acta Biol. Szeged. 46 (3/4) (2002) 63-65.

[60] L.J. Dai, Z.Q. Li, Comparative and functional genomics of wheat, Acta Bot. Boreal-Occident Sin. 24 (5) (2004) 949-953.

[61] R.L. Jing, X.P. Chang, Genetic diversity in wheat (T. aestivum) germplasm resources with drought resistance, Acta Bot. Boreal-Occident Sin. 23 (3) (2003) 410-416.

[62] J.X. Zhang, M.B. Kirham, Drought stress-induced changes in activities of superoxide dismutase, catalase, and peroxidase in wheat species, Plant Cell Physiol. 35 (5) (1994) 785-791.

[63] J.F. Gao (Editor-in-Chief), Experimental Technology in Plant Physiology, World Books Publishing Company, Xi'an, China, 2000.

[64] I.K. Vasil, The science and politics of plant biotechnology-a personal perspective, Nat. Biotechnol. 21 (8) (2003) 849-851.

[65] T.C. Hsiao, Plant responses to water stress, Ann. Rev. Plant Physiol. 24 (1973) 519-570.

[66] H.B. Shao, L.Y. Chu, Plant molecular biology in China: opportunities and challenges, Plant Mol. Biol. Rep. 23 (4) (2005) 345-358.

[67] H.B. Shao, L.Y. Chu, C.X. Zhao, et al., Plant gene regulatory network system under abiotic stress, Acta Biol. Szeged. 50 (1/2) (2006) 1-9. 